\title{
ARCHITECTURE OF A WEB-BASED POWER SCADA SYSTEM USING J2EE TECHNOLOGY
}

\author{
Qizhi Chen $^{1,2}$, Hamada Ghenniwa ${ }^{2}$, Weiming Shen ${ }^{2,3}$ \\ ${ }^{1}$ Southwest Jiaotong University, Chengdu, China \\ ${ }^{2}$ University of Western Ontario, London, Ontario, Canada \\ ${ }^{3}$ National Research Council Canada, London, Ontario, Canada \\ qzchen1625@gmail.com; hghenniwa@eng.uwo.ca; weiming.shen@nrc.gc.ca
}

\begin{abstract}
With the deregulation of the electrical power industry, there is a strong need for power SCADA system to interoperate with other information systems. The interoperation between SCADA and all other systems not only needs the information sharing, but also the business - or transaction-based information integration. This paper presents a Web-based architecture for power SCADA system, which is a multi-tier distributed framework using J2EE technology. This architecture combines pervasive browser with flexible user-specified application client, supports Web-service integration and transaction control, and provides a unified security policy. With the advantages of J2EE platform, this new Web-based SCADA system seamlessly integrates traditional SCADA with the enterprise information system, and exhibits more flexibility. scalability, and security than precious Web-browsing-only SCADA system.
\end{abstract}

\section{INTRODUCTION}

Supervisory control and data acquisition (SCADA) system plays an important role in a power system. It supports control of remote equipments, gathers data from substations and/or generation stations, and shares all the gathered information with other systems. From its appearance in 1960s to now, SCADA system has been developed from the first generation, mainframe-dominated, centralized computing system to the third generation, the distributed network computing system [1].

Because of the deregulation of electrical power industry, the combination of more dynamic energy markets and the growing importance of E-commerce is driving the greater needs of effective cooperation and information exchange or sharing among different systems or different electrical entities [2]. Because power SCADA system is the most important information resource for power system operation, maintenance as well as marketing, it faces the great challenge to provide information in a flexible, expandable, and standardized way to many other subsystems among the power information system, such as Electricity Trading System, Planning System, and Enterprise Information System (EIS). The rapid 
development of information technologies brings the great opportunity to the power SCADA system to deal with this challenge.

The widespread and successful Internet/Intranet and World Wide Web (WWW) technologies makes the new generation of Web-based power SCADA system possible. Various SCADA applications or sub-systems based on Web technologies have been addressed in the past [3-9]. But all these Web-based application simply aims on supplying the easy information access and lower client hardware investment.

This paper proposes a multi-tier distributed Web-based architecture for power SCADA system using Java 2 Platform, Enterprise Edition (J2EE). It combines pervasive browser client with flexible user-specified application client, supports Web-service integration and transaction control, and provides a unified security policy. With the advantages of J2EE platform, this new Web-based SCADA system seamlessly integrates traditional control- and monitoring-oriented SCADA with EIS, and exhibits higher flexibility, scalability, and security than precious Web-browsingonly SCADA system.

The remainder of this paper is organized as follows. The J2EE platform and related technologies are briefly introduced in Section 2. In Section 3, a multi-tier Web-based architecture for power SCADA system using J2EE technology is proposed. The software architecture of this Web-based SCADA system and its characteristics are discussed in Section 4. Section 5 gives a brief review of the related work. Conclusion and future work are included in Section 6.

\section{J2EE AND RELATED TECHNOLOGIES}

$\mathrm{J} 2 \mathrm{EE}$ is an architecture for distributed enterprise applications. It offers a multi-tiered distributed application model, the ability to reuse components, integrated Extensible Markup Language (XML)-based data interchange, a unified security, and flexible transaction control.

Application logic in J2EE is divided into components according to their function, and the various application components that make up a J2EE application are installed on different machines. The location of components depends on the tier to which the application component belongs. Four tiers are defined in J2EE platform, which are client tier, web tier, business tier, and enterprise information system (EIS) tier. The $\mathbf{J} 2 \mathrm{EE}$ application architecture is illustrated in Figure $\mathbf{1}$.

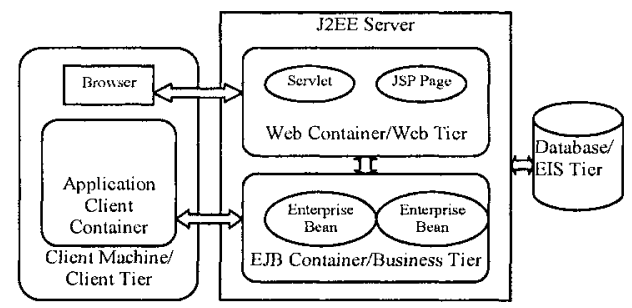

Figure 1 J2EE application architecture 
The J2EE specification defines client components, Web components, and business components as illustrated in Figure 1.

Client components: A J2EE client can be a Web client or Application client running on the client machine. A Web client contains dynamic Web pages generated by Web components running in the Web tier and a Web browser. An application client provides a way for users to handle tasks that require a richer user interface, typically a graphical user interface (GUI).

Web components: J2EE Web components are either Servlets or pages created using JavaServer Page technology (JSP pages). Servlets are Java programming language classes that dynamically process $h t t p$ requests and construct responses. JSP pages are text-based documents that execute as servlets but allow a more natural approach to create static content.

Business components: Business logic is handled by enterprise beans running in the business tier. An enterprise Java bean (EJB) receives data, process it, and send it back to client programs. An Enterprise Java Bean also retrieves data from or store data into the EIS tier. There are three kinds of enterprise beans in J2EE: session beans, entity beans, and message-driven beans.

In addition to all above characteristics, the most important and exiting improvement of the latest J2EE platform is substantial support for Web services, and WS-I Basic Profile 1.0 for Web services interoperability. This makes it possible to develop a Web-based application not only supporting Web pages sharing but also transaction-based information integration.

\section{SYSTEM FRAMEWORK OF WEB-BASED POWER SCADA SYSTEM}

The four-tier system framework of a Web-based power SCADA system is shown in Figure 2.

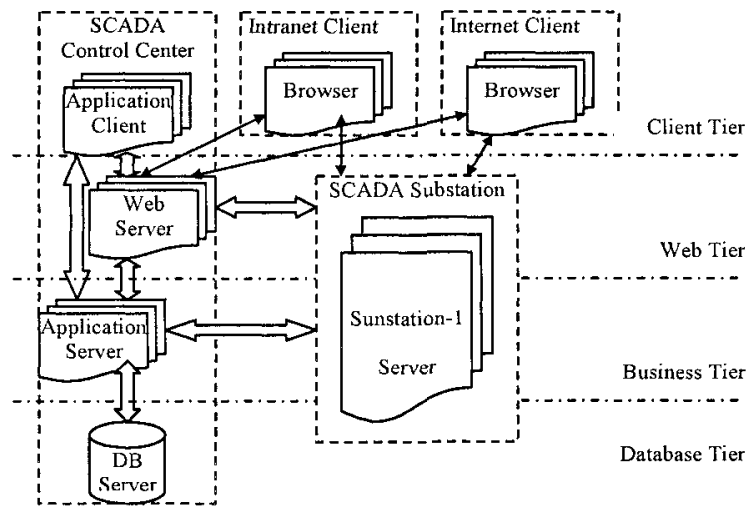

Figure 2 Four-tier framework of the Web-based power SCADA system using J2EE technology 
The system framework of this Web-based power SCADA system employs all four tiers defined in J2EE specification, which are client layer, Web layer, business layer, and database layer. In client layer, there are two different client types, one is standalone application client operated by SCADA operator in control center and another is standard thin browser without any plug-in used by Internet or Intranet users.

Each tier in the control center can be deployed into one or more computer nodes, which depends on the system scale, number of operator, and the reliability requirement. In order to keep the high reliability, the business tier and database tier are redundantly configured. The redundant application servers operate in parallel mode or conventional standby mode. The database servers are always configured with storage cluster. Because of the scalability of J2EE, the business layer and the database layer can be deployed into a same server in the case of a small-scale SCADA control center.

Instead of the direct connection to Intranet/Internet for each intelligent electrical device (IED) in a substation [7][9], the substation in Figure 2 is considered as an integrated web server or application server to connect to this Web-based SCADA system. The benefit of this is that no prerequisite to each IED that it must be webbased. Because of its size, the web layer, business layer, and database layer in a substation all be deployed into one physical machine.

The communication infrastructure of a Web-based power SCADA system is illustrated in Figure 3.

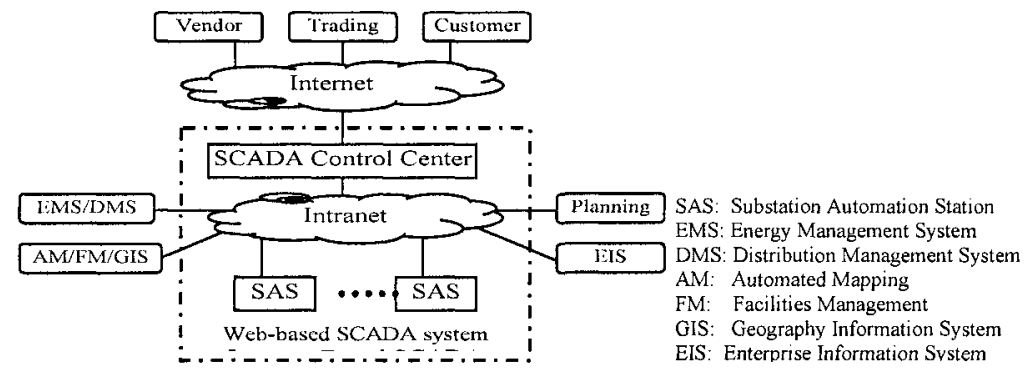

Figure 3 Communication infrastructure of the Web-based power SCADA system

Figure 3 shows the communication relationship between the Web-based SCADA system and other systems in a utility information system. It shows that the intraenterprise sub-systems, such as EMS, EIS, AM/FM/GIS, and Planning system, all connect with the Web-based SCADA through Intranet. The inter-enterprise subsystem, Trading system, as well as all extra-enterprise users, such as customers and vendors, access this Web-based SCADA system through Internet.

Since J2EE provide the Web services support through integrated data interchange on XML-based standard protocols, the access to SCADA system can be implemented through traditional Web page browsing or novel Web-services integration. 


\section{SOFTWARE ARCHITECTURE OF WEB-BASED POWER SCADA SYSTEM}

\subsection{Software Architecture}

This control center software architecture of the Web-based SCADA system using J2EE technology is illustrated in Figure 4. The software architecture for the substation server is similar with this except that all J2EE components all resides on the same server.

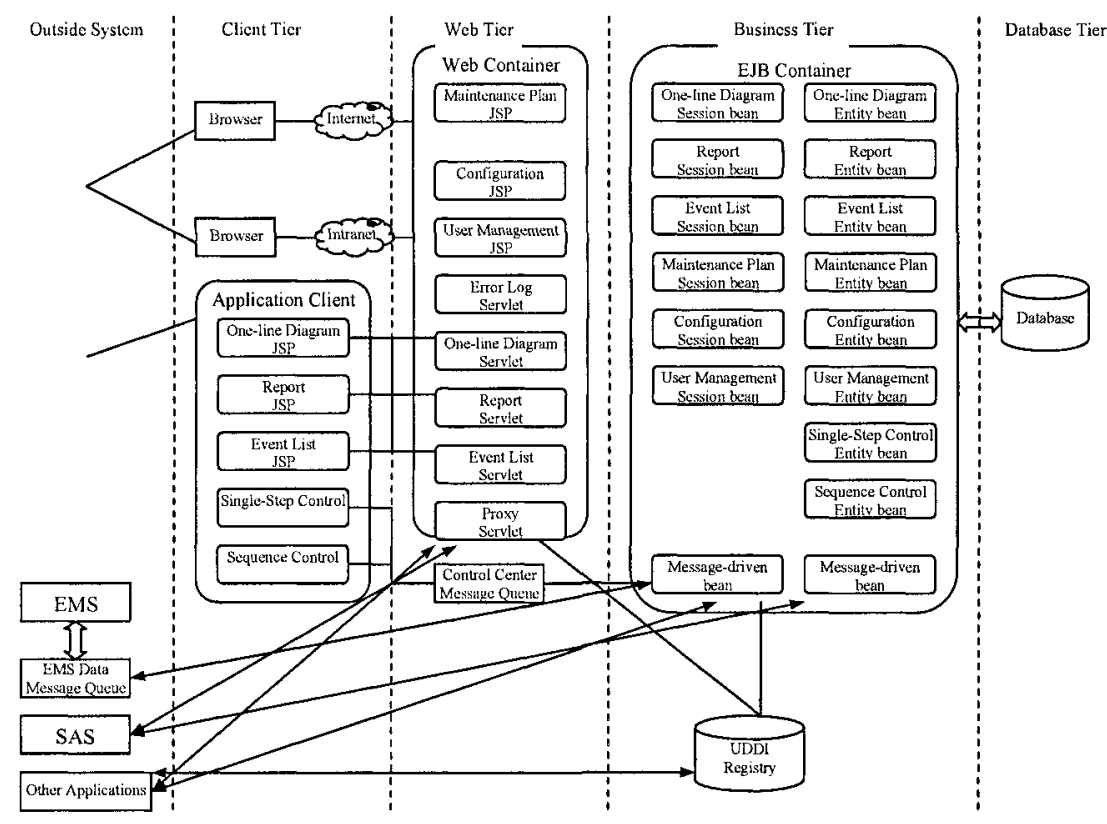

Figure 4 Control center software architecture of the Web-based SCADA system using J2EE technology

The key characteristics of this software architecture described in Figure 4 are:

The software architecture can be described as Client/Server (C/S) structure, but under the $\mathrm{C} / \mathrm{S}$ structure there are two different client components in this Web-based SCADA system. One is the prevalent thin Web browser, another is the independent user-specified application client. Internet or Intranet users use browser to get the real-time or history data, make maintenance plan, browse or modify system configuration, or trace system error logs. This allows a lot of widely distributed users can access the SCADA with no special application installation. The independent application client provides flexible and friendly man machine interface (MMI) to the SCADA operator. 
The main functions of application client are electrical equipment control, realtime or history information display. Except directly accessing the message-driven bean running in the business tier, the application client shares the several servlets running in the Web tier for information display with the Web client.

The Web tier includes both JSP pages and servlet components. JSP pages are used for its natural approach to creating static content, and servlets are used for the purpose that it can be accessed not only by browsers but also by application clients.

A proxy is implemented in the Web tier for two advantages. The first is that it insulates the SAS from the direct connection to the Internet. Proxy redirects all Internet accesses to SAS to their destination substation so as to reduce the access points to this Web-based SCADA system. Another advantage is that the Web tier in the control center does not need implement the same JSP if it has been deployed in a substation server.

The application client and EMS interact with message-driven bean running in the business tier through the JMS queues.

The control operation in SCADA is a three-step operation, which follows the order of selecting first, executing later, and certifying last. In order to ensure the data and control integrity, transaction control is employed for the SCADA equipment control operation.

SAS has the similar software architecture as the control center except that all J2EE components are deployed into one machine. SAS communicates with message-driven bean running on the $\mathrm{J} 2 \mathrm{EE}$ server in the control center through the TCP-IP based standard protocols, such as IEC60870-5-104. In the mean time, it provides the dynamic Web contents to Intranet through JSP pages or sevlets.

To those interfaces which are need to be open among the SCADA or to other systems, they will be encapsulated as Web services and their corresponding Web Service Description Language (WSDL) file will be published to the Universal Description Discovery \& Integration (UDDI) registry. Thus every application that has the right to access the SCADA system can find the Web services and using it. The endpoint of Web services in this system can be a JAX_RPC Web tier component or session bean. Service endpoint.

\subsection{System Security}

The security implementation in this Web-based distributed SCADA system only adopts the declarative security in J2EE specification. Different security groups such as operator, maintenance engineer, manager, custom, and vendor, are defined. Each group owns different permission to access the SCADA resources and includes a set of authenticated users. For example, the operator group can access the information display Web components, and control EJB, but it does not have the right to access system configuration Web components.

The other security policy adopted by this Web-based SCADA system is that SAS does not directly connect to Internet but all Internet accesses to it are forwarded by the proxy in the control center. This greatly reduces the Internet access point to the SCADA so as to reduce the possibility of being attacked by malicious users. 


\section{RELATED WORK}

There are significant research efforts focused on proposing Web-based SCADA for power system, which covers the sub-system in SCADA, intelligent electric device (IED), and dependent applications in SCADA system. The IT technologies employed by these works vary from Java, ASP, Applet to J2EE. The followings are brief reviews of some related research efforts.

Two Web-based sub-systems in power SCADA system were discussed in [3]-[4]. The work of Leou et al. [3] described a Web-base 3-tier power quality monitoring system, which used ASP technology to connect to and read from database server, created dynamic html pages. Compared with our proposal, it only implemented the Web client and did not support Web-service interface. The work of Qiu et al. [4] introduced an Internet based frequency monitoring network. It emphasized the Internet as the physical communication infrastructure more than the Internet-based software implementation.

The work of Qiu et al. [5] implemented a Web-based SCADA display application via Internet access, which was a typical three-tier client/server applications containing a service tier, a data store tier, and a Man Machine Interface (MMI) tier. The MMI was implemented by Java language without using any advanced Web technologies. Compared with our full implementation of SCADA system, the WWW displays in this system only limited to the tabular displays and one-line diagrams.

Some issues about Web-based power SCADA system were discussed in [7]-[9]. Ebata et al. in [7] used a trial system to discuss how to improve the real-time performance and reliability of Internet-based SCADA, but it did not discuss the technology implementation, which is the emphasis of our paper. The work of Medida et al. [8] just employed a gateway as Web server in the conventional SCADA center in order to support information browsing. It just was a temporary solution for the SCADA information sharing and not an ultimate solution as ours. The work of Li et al. [9] regards the SCADA control center system and individual intelligent devices (IED) as separate Web-based application. Compared with our proposed architecture, it treated individual IEDs as separate Web components. The shortcoming is that this imposes a prerequisite to each IED, and SCADA system will loose the control if it is not a Web-based device.

Although two systems based on J2EE platform in power system were discussed in [11][12], [11] aimed on a simple power quality monitoring system and [12] was about the electricity market operation system, which were all different from the SCADA system.

\section{CONCLUSION AND FUTURE WORK}

The proposed Web-based architecture for power SCADA system is a multi-tier distributed framework using J2EE technology. This architecture combines pervasive browser client with flexible user-specified application client. Since the browser implemented by Java Servlet or JSP has not any needs for Java Plug-in or any possible security policy in the client site, it makes the browser thinner than any of 
previous implements using Applets for dynamic Web content. On the other hand, the standalone application client provides more flexible user-specified GUIs to the operator than just using marked language-based pages.

With the advantages of J2EE technology, this new Web-based power SCADA architecture facilitates transaction control, Web-service support, a unified security policy, and exhibits higher flexibility, scalability, and security. It makes power SCADA system easily achieve the information sharing as well as the business logicand transaction-based information integration with other systems.

Future works will include implementing a prototype system, design the policy that which kind of services in SCADA should be published as Web services, and search the possible solution to improve the real-time response capability caused by the lower efficiency of Java.

\section{REFERENCE}

1. McClannhan, RH. "SCADA and IP: Is Network Convergence Really Here?", IEEE Industry Applications Magazine, 2003; 9(2): 29-36.

2. Becker, D, Falk, H, Gillerman, J, Mauser, S, Podmore, R, Schneberger, L. "Standards-Based Approach Integrates Utility Applications", IEEE Computer Applications in Power, 2000; 13(4): 13-20

3. Leou, RC, Chang, YC, Teng, JH. "A Web-based power quality monitoring system", Power Engineering Society Summer Meeting, 2001, IEEE, Vol. 3, pp. 1504-1508.

4. Qiu, B, Chen, L, Centeno, V, Dong, X, Liu, Y. "Internet based frequency monitoring network (FNET)", Power Engineering Society Winter Meeting, 2001. IEEE, Vol. 3, pp.1166-1171.

5. Qiu, B, Gooi, HB. "Web-Based SCADA display systems (WSDS) for access via Internet", Power Systems, IEEE Transactions on, , 2000, 15(2): 681-686.

6. Ishihara, Y, Shirota, Y, Sekiguchi, K, Sato, S, Sawai, K. "Recent trends in the implementation of Intranet based measurement and monitoring", Transmission and Distribution Conference and Exhibition 2002: Asia Pacific. IEEE/PES, Vol. 3, pp. 2261-2266, 2002.

7. Ebata, Y, Hayashi, H, Hasegawa, Y, Komatsu, S, Suzuki, K. "Development of the Intranet-based SCADA (supervisory control and data acquisition system) for power system", IEEE PES Winter meeting 2000, Vol. 3, pp. 1656-1661, 2000.

8. Medida, S, Sreekumar, N, Prasad, KV. "SCADA-EMS on the Internet", Energy Management and Power Delivery, 1998. Proceedings of EMPD '98. 1998 International Conference on, Vol. 2, pp. 656-660, 1998.

9. Li, D, Serizawa, Y, Kiuchi, M. "Concept Design for a Web-based Supervisory Control and DataAcquisition (SCADA) System", Transmission and Distribution Conference and Exhibition 2002: Asia Pacific, ILEE/PES, Vol. 1, pp. 32-36, 2002.

10. Bodoff, S, Armstrong, E, Ball, J, Carson, DB, Evans, I, Green, D, Haase, K, Jendrock, E. "The J2EE Tutotial: Second Edition", Sun Microsoft, 2004.

11. Leou, RC, Tsai, WR, Chang, YN. "A Power Quality Monitoring System Based on J2EE Architecture", TENCON 2004, 2004 IEEE Region 10 Conference, Vol. C, pp. 291-294, 2004.

12. Song, $Y$, Tang, G, Yang, Z, Hu, J. "The Technical Implementation of the Electricity Market Operation System", Transmission and Distribution Conference and Exhibition: Asia and Pacific, $2005 \mathrm{IEEL} / \mathrm{PES}$, pages:6, 2005. 